Article

\title{
Quaternions and Functional Calculus
}

\author{
Elham Ghamari * and Dan Kučerovský \\ Department of Mathematics and Statistics, University of New Brunswick, Fredericton, NB E3B 5A3, Canada \\ * Correspondence: eghamari@unb.ca
}

Received: 29 May 2019; Accepted: 18 July 2019; Published: 26 July 2019

check for

updates

\begin{abstract}
In this paper, we develop the notion of generalized characters and a corresponding Gelfand theory for quaternionic $C^{*}$-algebras. These are $C^{*}$-algebras whose structure permits an action of the quaternions. Applications are made to functional calculus, and we develop an S-functional calculus related to what we term structural regular functions.
\end{abstract}

Keywords: quaternions; hilbert space; functional calculus; gelfand theorem; $\mathrm{C}^{*}$-algebras

MSC: Primary 46A32 46L06; Secondary 47L07; 47L50

\section{Introduction}

The main point of our work is to extend a theorem that is generally agreed to be only valid for the commutative case (the Gelfand theorem) of a certain class of noncommutative $C^{*}$-algebras. The class in question is defined using quaternions, and the main applications of our theorem involve functional calculus. We now introduce these two topics.

In this section, we introduce functional calculus, and then in the next section we introduce quaternionic $C^{*}$-algebras. Let $X$ be a finite-dimensional $C^{*}$-algebra over the field of complex numbers $\mathbb{C}$. If $T \in X$ is a normal linear operator, then as is well known, a functional calculus can be defined with the standard eigenvalue definition, eigenvalues being the values $\lambda \in \mathbb{C}$ for which $T-\lambda I$ is not invertible. When considering quaternonic $C^{*}$-algebras, new difficulties arises because of the lack of commutativity of the quaternions. Indeed, we will see that there are two different eigenvalue problems. Additional problems arise if we want to drop finite dimensionality. We discuss first the problems arising from dropping finite dimensionality and then return to the problems arising from non-commutativity.

Consider a one-sided polynomial $P(x)=a_{n} x^{n}+a_{n-1} x^{n-1} \ldots$, with coefficients in the quaternions $\mathbb{H}$, and a $C^{*}$-algebra $A$ over $\mathbb{H}$. The elements of the $C^{*}$-algebra are right linear with respect to quaternion scalars. If we regard the formal variable $x$ as commuting with its coefficients, then these one-sided polynomials form a ring. We can apply $P(x)$ to an element $T$ of the $C^{*}$-algebra. Thus, the application map $P \mapsto P(T)$ takes a polynomial to an element of the $C^{*}$-algebra $A$. This is an example of the so-called functional calculus. The well-known von Neumann inequality [1] shows that the application map is continuous and norm decreasing provided only that the element $T$ belongs to the unit ball of the $C^{*}$-algebra.

It is natural to wonder if by continuity or otherwise, one could consider extending the construction to a larger class of functions. There are several possible ways this can be done, for example, slice regularity [2], the theory of slice hyperholomorphic functions, the related $S$-functional calculus, and Riesz-Dunford functional calculus for bounded operators [3]. In another direction, one can put conditions on the operator, such as normality, or conditions on the $\mathrm{C}^{*}$-algebra, leading, for example, to the spectral theorem for normal operators for quaternionic von Neumann algebras [3]. 


\section{The Basics of $C^{*}$-Algebras, or Why Are $C^{*}$-Algebras Beautiful?}

As is well-known, Hilbert space is the canonical example of an infinite-dimensional Euclidean space. Since Hilbert space is perfectly symmetrical and the theory of Hilbert space can be developed in a very perfect form, most of the interest of Hilbert space has to do with the operators and operations on it. $C^{*}$-algebras are norm-closed algebras of operators on a Hilbert space. Thus, they have a sort of symmetry property, as a consequence of the $\mathrm{C}^{*}$-equation explained below, that relates their norm (which is induced from the Hilbert space) and the so-called spectrum of their elements. General Banach algebras, or for that matter, Banach spaces, have nothing like this. Since, furthermore, Hilbert space is isomorphic to its own dual, due to the Riesz representation theorem, many operations that in Banach algebra/Banach space theory would lead to maps into the dual will, in the $C^{*}$-case, give self-mappings of the $C^{*}$-algebra. The Hilbert space inner product provides an adjoint operation that is usually written *, and thus if $T$ is an element of some $C^{*}$-algebra, there exists an adjoint operator $T^{*}$ that satisfies the Hilbert space property $\langle x, T y\rangle=\left\langle T^{*} x, y\right\rangle$, and $T^{*}$ is in the given $C^{*}$-algebra. This is a further example of how intrinsically symmetrical $C^{*}$-algebras are. Thus, $C^{*}$-algebras can be considered as Banach algebras with a $*$-involution, or as so-called $B^{*}$-algebras. (They are also thus Banach spaces.) At this point, the theory begins to diverge somewhat according to whether we take the scalar field of the Hilbert space to be the real numbers, the complex numbers, or the noncommutative quaternions. In the complex case, the $C^{*}$-algebras are distinguished amongst the $B^{*}$-algebras by the famous $C^{*}$-equation:

$$
\left\|x x^{*}\right\|=\|x\|^{2} .
$$

We are primarily interested here in the quaternion case, but we remark that the quaternion case is a subclass of the real case if we simply forget the quaternionic structure and use the real subset of the quaternions as the scalar field. Quaternionic $C^{*}$-algebras could be regarded as $\mathrm{B}^{*}$-algebras with quaternionic structure added, and then the key property that distinguishes the $C^{*}$-algebras within this larger class is again the above $\mathrm{C}^{*}$-equation, supplemented by the additional condition that

$1+x^{*} x$ is invertible in the unitization of $A$ for any element $x \in A$.

Thus, real $C^{*}$-algebras will have a slightly different theory than complex $C^{*}$-algebras.

Although $C^{*}$-algebras by themselves do not have enough structure to have a fully developed theory of harmonic analysis, commutative real or complex $C^{*}$-algebras have such a property, the well-known Gelfand theorem. (Note that quaternionic $C^{*}$-algebras are hardly ever commutative because the quaternions are not commutative.) One form of the theorem is:

Theorem 1 (Gelfand [4]). A commutative unital real or complex $C^{*}$-algebra A has sufficiently many characters to separate elements. Furthermore, the map $\pi: A \rightarrow C(X)$, where $C(X)$ denotes the continuous functions on the characters $X$, is a $C^{*}$-isomorphism.

The characters together with a natural topology, denoted by $X$, in the above theorem, are often referred to as the Gelfand spectrum. The topology is the weak* topology (or product topology) defined by evaluating the elements of $X$ on elements of the $C^{*}$-algebra. It is known that the Gelfand spectrum $X$ is compact when the given $C^{*}$-algebra is unital. To simplify our arguments, we will focus henceforth on the unital case. The Gelfand theorem doese not apply to the quaternionic case for the fundamental reason that quaternionic $C^{*}$-algebras are almost never commutative (see, however, [5], Theorem 4.10). One of our main ideas is to therefore accommodate this lack of commutativity by weakening the definition of a character:

Definition 1. A unital *-homomorphism $h: A \rightarrow \mathbb{H}$ will be called a generalized character on the quaternionic $C^{*}$-algebra $A$. 
We will soon show that there is a plentiful supply of such characters in at least one important case: the quaternionic sub-C*-algebra generated by a normal operator; we will moreover establish a Gelfand-type theorem, and we will compute the (generalized) Gelfand spectrum in terms of more concrete objects. We now complete the picture by giving some definitions that are needed for a solid description of quaternionic $C^{*}$-algebras but that were omitted from the introduction to make it flow more smoothly.

Definition 2. 1. $\quad X$ is a $\mathbb{H}-$-vector space or quaternionic two sided vector space if $X$ is a vector space with respect to the right scalar multiplication $X \times \mathbb{H} \ni(x, q) \mapsto x q \in X$ and is also a vector space with respect to the left scalar multiplication $\mathbb{H} \times X \ni(q, x) \mapsto q x \in X$.

2. Let $X$ be a $\mathbb{H}$-vector space, then $X$ is called a $\mathbb{H}$-algebra if the following conditions hold:

$$
q(x y)=(q x) y,(x q) y=x(q y), \text { and }(x y) q=x(y q)
$$

for every $x, y \in X$ and $q \in \mathbb{H}$.

3. A $\mathbb{H}$-involution on $X$ is a self-map $a \mapsto a^{*}$ of $a \mathbb{H}$-algebra $X$ such that

$$
x^{* *}=x,(q x)^{*}=x^{*} q^{*}, \text { and }(x q)^{*}=q^{*} x^{*}
$$

for every $x, y \in X$ and $q \in \mathbb{H}$. Then $X$ is called $a \mathbb{H}$-involutive algebra.

4. Let $X$ be a $\mathbb{H}$-involutive algebra that is equipped with a $\mathbb{H}$-norm $\|$.$\| . Then X$ is called a $\mathbb{H}-C^{*}$-algebra if it is complete under the norm and satisfies

$$
1+x^{*} x \text { is invertible in the unitization of } A \text { for any element } x \in \text { Aand }\left\|x^{*} x\right\|=\|x\|^{2} .
$$

We now give routine definitions of right linearity and of boundedness.

Definition 3. Let $X$ be a $\mathbb{H}-C^{*}$-algebra. A map $T: X \rightarrow X$ is said to be right linear if

$$
T(x q+y)=T(x) q+T(y)
$$

for all $q \in \mathbb{H}$ and $x, y \in X$. We say that $T$ is bounded if there exists $M>0$ such that $\|T x\| \leq M\|x\|$, for all $x \in X$. Equivalently, if $T$ is bounded, then

$$
\|T\|:=\sup \{\|T x\|: x \in X,\|x\|=1\}
$$

is finite.

Definition 4. Let $T$ be a bounded operator on $X$. Then $T$ is said to be normal if $T^{*} T=T T^{*}$.

We now define left and right eigenvalues; this distinction between left and right arises from the noncommutativity of the quaternions.

Definition 5. Let $T$ be a right linear operator on a $\mathbb{H}-C^{*}$-algebra $X$. A quaternion $\lambda \in \mathbb{H}$ is called a left eigenvalue of $T$ if there exists a vector $x \in X \backslash\{0\}$ such that

$$
T(x)=\lambda x .
$$

And $\lambda$ is called a right eigenvalue if if there exists a vector $x \in X \backslash\{0\}$ such that

$$
T(x)=x \lambda
$$


Indeed, if $\lambda$ is a right eigenvalue, the sphere $q^{-1} \lambda q, q \in \mathbb{H} \backslash\{0\}$ consists of eigenvalues. If $\lambda$ is a left eigenvalue, $T-\lambda I$ is right linear; however, this eigenvalue has unexpected properties in the case of self-adjoint operators. To generalize the notions of eigenvalues to a notion of spectrum leads to two natural situations: $\lambda$ is said to be in the left spectrum of $T$ if $T-\lambda I$ is not invertible, and $\lambda$ is in the right spectrum if $T-I \lambda$ is not invertible.

\section{Gelfand Theory with Generalized Characters}

As previously explained, the Gelfand theorem applies to real or complex commutative $C^{*}$-algebras, taken to be unital for simplicity, but the Gelfand theorem does not apply to the quaternionic case for the fundamental reason that $\mathbb{H}-C^{*}$-algebras are almost never commutative. Given a normal operator $T$ in a $\mathbb{H}-C^{*}$-algebra, we now consider generalized characters on $C_{\mathbb{H}}^{*}(1, T)$, the $\mathbb{H}$-sub $-C^{*}$-algebra generated by $T$ and 1 . Recall that a generalized character of the $\mathbb{H}-C^{*}$-algebra $A$ is a unital $*$-homomorphism $h: A \rightarrow \mathbb{H}$. There is a natural equivalence relation on the set of generalized characters:

Definition 6. Two generalized characters $h_{1}$ and $h_{2}$ on a quaternionic $C^{*}$-algebra $A$ are equivalent if there is a quaternion $q$ such that $h_{1}(x)=q h_{2}(x) q^{-1}$, where $\bar{q}=q^{-1}$, for all $x$ in the $C^{*}$-algebra $A$.

The above definition has no counterpart in the real or complex case, as it uses in a basic way the noncommutative nature of the quaternions.

Definition 7. The Gelfand space of a quaternionic $C^{*}$-algebra is the space of generalized characters modulo equivalence.

We say that a unital quaternionic $C^{*}$-algebra $A$ is of the Gelfand type if it is *-isomorphic to $C(X, \mathbb{H})$, where $X$ is its Gelfand space, and $C(X, \mathbb{H})$ is the $C^{*}$-algebra of continuous quaternion-valued functions on $X$. We postpone the discussion of the topology on the generalized Gelfand space to after Lemma 3. The two natural questions that arise are: What $C^{*}$-algebras are of the Gelfand type? And in what cases can we describe the Gelfand space in terms of simpler objects?

Theorem 2. Let $X$ be $a \mathbb{H}-C^{*}$-algebra. The real part of $X$ is defined by

$$
X_{R}:=\{x \in X: \alpha x=x \alpha \text { for any } \alpha \in \mathbb{H}\}
$$

is a real $C^{*}$-algebra, and $X_{R} \otimes \mathbb{H}$ is isomorphic to $X$.

Lemma 1. Let $h_{1}$ and $h_{2}$ be two generalized characters on a quaternionic $C^{*}$-algebra, A. The following are equivalent.

1. The characters have the same kernel.

2. $\quad$ The characters are equivalent in the sense of Definition 6.

3. The characters are equal when restricted to the real part subalgebra $A_{R}$ of $A$.

Proof. We show that (1) implies (2). If the kernels of $h_{1}$ and $h_{2}$ are equal, then a routine diagram chase in the following diagram defines a map $\alpha: \mathbb{H} \rightarrow \mathbb{H}$.

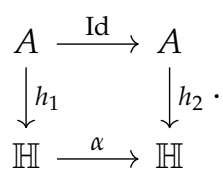

The map $\alpha: \mathbb{H} \rightarrow \mathbb{H}$ is, at the algebraic level, a *automorphism of $\mathbb{H}$. Such automorphisms are always continuous, since $\mathbb{H}$ is, after all, a $C^{*}$-algebra. But since $\mathbb{H}$ is simple and has finite dimension over its center, $\mathbb{R}$, the Skolem-Noether theorem implies that the automorphism $\alpha$ is inner and has the form $x \mapsto a x a^{-1}$. Since $\alpha$ is also a *automorphism, we deduce the additional condition that $a^{-1}=\bar{a}$. 
This makes $h_{1}$ and $h_{2}$ equivalent in the sense of Definition 6, as claimed. Now we show that (2) implies (3). If we restrict $h_{1}$ and $h_{2}$ to the real part $A_{R}$ of $A$, then they become real valued, and as the only ring automorphism of $\mathbb{R}$ is the identity, the automorphism implementing the equivalence of $h_{1}$ and $h_{2}$ becomes trival after this restriction. Thus, if we restrict $h_{1}$ and $h_{2}$ to the real part $A_{R}$ of $A$, they become equal, as claimed. Now we show that (3) implies (1): from the condition (3), the real parts of the kernels of $h_{1}$ and $h_{2}$ are equal, but since a kernel is, after all, a quaternionic $C^{*}$-algebra, the kernels are equal if their real parts are equal.

The above lemma provides a one-to-one algebraic correspondence between the points of the Gelfand space of a quaternionic $C^{*}$-algebra and the characters of the real part of the $C^{*}$-algebra. The topology on our Gelfand space has not yet been specified. Let us take the topology to be such that this algebraic correspondence becomes a homomorphism. Now we can prove our most general Gelfand-type theorem. There are related results in $[5,6]$, but in those theorems, the Gelfand space is an abstract topological space. In our case, we provide a description in terms of characters, just as in the classic Gelfand theorem (Theorem 1).

Theorem 3. A unital quaternionic $C^{*}$-algebra $A$ is of the Gelfand type if and only if all operators in the $C^{*}$-algebra are normal. If $A$ is of the Gelfand type, then there exist sufficiently many generalized characters to separate elements. Furthermore, the map $\pi: A \rightarrow C(X)$, where $C(X)$ denotes the continuous quaternion-valued functions on the Gelfand space X, is a $C^{*}$-isomorphism. The Gelfand space X consists of the generalized characters, after equivalence.

Proof. It is clear that if the $C^{*}$-algebra is of the Gelfand type and is hence isomorphic to $C(X, \mathbb{H})$, then the $C^{*}$-algebraic involution is isomorphic to the quaternionic conjugation, applied pointwise, in $C(X, \mathbb{H})$. However, the quaternions have the remarkable algebraic property that $\bar{q} q=q \bar{q}$. Thus, all elements of a Gelfand-type algebra are normal. For the opposite direction, take the real part of $A$. This is a real $C^{*}$-algebra where all operators are normal, and in the real case the polarization identity then implies commutativity. Then Theorem 1 , this real $C^{*}$-algebra $A_{R}$ is isomorphic to $C_{R}(X)$, the algebra of real functions on the space of characters $X$ of $A_{R}$. By Lemma 1, the space $X$ can be identified with the generalized character Gelfand space of $A$. Then, tensoring by $\mathbb{H}$, we find that $A$ is isomorphic to $C(X, \mathbb{H})$.

Our main Gelfand-type result for this situation is:

Theorem 4. If $T$ is a quaternionic normal operator, $C_{\mathbb{H}}^{*}(1, T) \cong C\left(\sigma_{L}(T), \mathbb{H}\right)$.

Proof. Let $A=C_{\mathbb{H}}^{*}(1, T)$. Let us first dispose of a special case. Let $h$ be a nonzero generalized character of $A$. If it has no kernel, then since the range must be all of $\mathbb{H}$, it follows that in fact $C_{\mathbb{H}}^{*}(1, T) \cong \mathbb{H}$, and so $T$ is a scalar multiple of 1 . In this case, the result is trivially true. If, on the other hand, $A$ is not isomorphic to $\mathbb{H}$, then every generalized character has a nontrivial kernel. Let $h$ be a generalized character. If $h(T)=0$, then since also $h(1)=1$, it follows that $h$ is the trivial character. If, on the other hand, $h(T) \neq 0$, then there exists a $\lambda \in \mathbb{H}$ such that $h(T)=\lambda$. Therefore, $T-\lambda i d$ is in ker $h$. Note that if $v \in A$ is invertible, then $h(v) \neq 0$, and thus, in particular, it follows that $T-\lambda i d$ is not invertible. But this means that $\lambda$ is in the spectrum $\sigma_{L}(T)$. Let us now show that for a given character $h$, there is only one $\lambda$ such that $T-\lambda i d$ is in ker $h$. Suppose not. Thus, $T-\lambda_{1} i d$ and $T-\lambda_{2} i d$ are both in $\operatorname{ker} h$. Then $h\left(\left(\lambda_{1}-\lambda_{2}\right) i d\right)=0$, and since $h$ is a unital ${ }^{*}$-homomorphism, this implies that $\lambda_{1}=\lambda_{2}$. Evidently, after equivalence, each generalized character is determined by its kernel, and we have shown that each such kernel contains one and only one element of the form $T-\lambda i d$, where necessarily $\lambda$ is in the spectrum $\sigma_{L}(T)$. It remains to show that every possible value of $\lambda$ in $\sigma_{L}(T)$ does in fact appear; in other words, that given $\lambda$ in the spectrum $\sigma_{L}(T)$, there exists a generalized character $h$ such that $T-\lambda i d$ is in ker $h$. If this were not the case, then this would mean that under the isomorphism $A \cong C(X, \mathbb{H})$ provided by Theorem 3, the element $(T-\lambda 1) \in A$ corresponds to a function $f \in C(X, \mathbb{H})$ that is 
not zero at any generalized character $x \in X$. But then, since $X$ is compact and $\mathbb{H}$ is a division ring, it follows that $f$ is invertible in $C(X, \mathbb{H})$. This would mean that $T-\lambda 1$ is invertible in $A$, which is not the case. Thus, we may consider the map $\phi: X \rightarrow \sigma_{L}(T)$, which takes each character into the unique $T-\lambda i d \in \operatorname{ker} h$, where $X$ is the set of generalized characters on $A$. It is straightforward to check that if two characters are close in norm, then the corresponding values of $\lambda$ are close in norm, and conversely. Thus, this map is a homeomorphism. Hence, $X \cong \sigma_{L}(T)$, and so $C_{\mathbb{H}}^{*}(1, T) \cong C(X, \mathbb{H})$, as claimed.

There are results showing that simple $C^{*}$-algebras are surprisingly often finitely or singly generated. Thus, it is possibly interesting to consider when our (non-simple) class of Gelfand-type $C^{*}$-algebras could be finitely generated:

Theorem 5. $A=C(X, \mathbb{H})$ is finitely generated if and only if $X \subset \mathbb{H}^{n}$ for some $n$.

Proof. Suppose that $X \subset \mathbb{H}^{n}$. Then the coordinate functions of $\mathbb{H}^{n}$ provide a finite set of generators for $C(X, \mathbb{H})$. On the other hand, if $A$ is finitely generated, let the generators be $a_{1}, \ldots, a_{n}$. These generators are normal, and the continuous functional calculus gives injections $C\left(\sigma_{L}\left(a_{i}\right)\right) \rightarrow A$. Dualizing, we obtain surjections $P_{i}: X \rightarrow \sigma_{L}\left(a_{i}\right)$, and thus we have a (necessarily injective) map $X \rightarrow \prod_{i=1}^{N} \sigma_{L}\left(a_{i}\right)$ where the right hand side is the topological product of finitely many closed compact subsets of $\mathbb{H}$. But then $X \subset \mathbb{H}^{n}$, as claimed.

\section{S-Functional Calculus}

Now let us recall that the spectrum of a bounded complex linear operator $T$ acting on a complex $C^{*}$-algebra $X$ is defined by

$$
\sigma(T)=\{\lambda \in \mathbb{C}: T-\lambda i d \text { is not invertible }\},
$$

and then for $\lambda$ in the resolvent set $\rho(T):=\mathbb{C} \backslash \sigma(T)$, the resolvent $(T-\lambda i d)^{-1}$ is a holomorphic function with values in $B(X)$, the set of all bounded linear operators on a $C^{*}$-algebra $X$ endowed with the natural norm. In the quaternionic case, we have the real spectrum defined by a real representation, left and right quaternionic spectrum, and also slice spectrum, which is defined by

$$
\sigma_{s}(T)=\left\{s \in \mathbb{H}: T^{2}-2 \operatorname{Re}(s) T+|s|^{2} i d \text { is not invertible }\right\} .
$$

And then the S-resolvent set of $T$ is defined by $\rho_{S}(T):=\mathbb{H} \backslash \sigma_{\mathcal{S}}(T)$.

Definition 8. Let $T \in B(X)$ be a bounded right linear operator and $s \in \rho_{s}(T)$. We define the left S-resolvent operator

$$
S_{L}^{-1}(s, T)=-\left(T^{2}-2 \operatorname{Re}(s) T+|s|^{2} i d\right)^{-1}(T-\bar{s} i d) .
$$

Definition 9. Let $\Omega \subset \mathbb{H}$ be an open set, and let $f: \Omega \rightarrow \mathbb{H}$ be a real differentiable function. Let $I \in \mathbb{S}$, where $\mathbb{S}$ is the sphere of unit imaginary quaternions

$$
\mathbb{S}:=\left\{I=i x_{1}+j x_{2}+k x_{3}: x_{1}^{2}+x_{2}^{2}+x_{3}^{2}=1\right\}
$$

and $f_{I}$ is the restriction of $f$ to the complex plane $\mathbb{C}_{I}:=\mathbb{R}+I \mathbb{R}$. Then $f$ is a left regular function if for every $I \in \mathbb{S}$,

$$
\frac{1}{2}\left(\frac{\partial}{\partial x}+I \frac{\partial}{\partial y}\right) f_{I}(x+I y)=o
$$

In the above known definition, a choice is being made, because clearly there are different choices of imaginary unit $I$ that could be made. We will show the new result that there is a form of independence of choice (Corollary 1 below.) 
Definition 10. (S-functional calculus) Let $T \in B(x)$, and $f$ be a left regular function. We define

$$
f(T)=\frac{1}{2 \pi} \int_{\Omega} S^{-1}(s, T) d s_{I} f(s),
$$

where $\Omega$ is an open bounded set that contains $L_{I} \cap \sigma_{s}(T)$.

Now let $h=h_{0}+i h_{1}+j h_{2}+k h_{3}$. We define the conjugacy class of $h$ by $[h]$, all $h^{\prime}=h_{0}^{\prime}+i h_{1}^{\prime}+$ $j h_{2}^{\prime}+k h_{3}^{\prime}$ such that there is $a \in \mathbb{H} \backslash\{0\}$ for which we have $h^{\prime}=a h a^{-1}$, which is equal to the 2 -sphere

$$
[h]=\left\{h^{\prime} \in \mathbb{H}: \operatorname{Re}(h)=\operatorname{Re}\left(h^{\prime}\right) \text { and } h_{1}^{2}+h_{2}^{2}+h_{3}^{2}=h_{1}^{\prime 2}+h_{2}^{\prime 2}+h_{3}^{\prime 2}\right\} .
$$

Theorem 6. Let $h \in \sigma_{s}(T)$. If $h^{\prime} \in[h]$, then $h^{\prime} \in \sigma_{s}(T)$. Conversely, if $h, h^{\prime} \in \sigma_{s}(T)$, then there is $a \in \mathbb{H} \backslash\{0\}$ such that $h^{\prime}=a h a^{-1}$.

Proof. Let $h \in \sigma_{s}(T)$, which means $T^{2}-2 \operatorname{Re}(h) T+|h|^{2} i d$ is not invertible. But because $h^{\prime} \in[h]$, $\operatorname{Re}\left(h^{\prime}\right)=\operatorname{Re}(h)$ and $|\operatorname{Im}(h)|=\left|\operatorname{Im}\left(h^{\prime}\right)\right|$, thus $T^{2}-2 \operatorname{Re}\left(h^{\prime}\right) T+|h|^{2} i d$ is not invertible. On the other hand, let $h \in \mathbb{C}_{I}$ and $h^{\prime} \in \mathbb{C}_{J}$ be in $\sigma_{S}(T)$, where $I, J \in \mathbb{S}$. Then, as before, there exists an inner *-automorphism $\sigma: \mathbb{H} \rightarrow \mathbb{H}$, defined by $h \mapsto a h a^{-1}$, which takes $\mathbb{C}_{I}$ into $\mathbb{C}_{J}$, which means that $h^{\prime} \in[h]$. Moreover, $\sigma$ is unitary. Let $a \in \mathbb{H}$. Then consider the polar decomposition $a=|a| u$, where $u$ is unitary, i.e., $u^{*} u=u u^{*}=1$, where $u \in \mathbb{H}$ and $*$ is the conjugation. Thus,

$$
\sigma(h)=|a| u h u^{*}|a|^{-1}=u h u^{*},
$$

but $\left(u h u^{*}\right)\left(u h u^{*}\right)^{*}=1$.

Corollary 1. Let $T$ be a bounded right linear operator on a two-sided $\mathbb{H}-C^{*}-$ algebra, and let $f: \mathbb{H} \rightarrow \mathbb{H}$ be a continuous function on $\sigma_{s}(T)$. Let us consider two different choices of imaginary unit $I_{1}$ and $I_{2}$ in the slice functional calculus. If we denote by $f_{1}(T)$ and $f_{2}(T)$ the two operators obtained by using the slice functional calculus with each of these two slices, then there is $a \in \mathbb{H} \backslash\{0\}$ such that $f_{1}(T)$ is isomorphic to $f_{2}(T) a^{-1}$.

Proof. Choose an automorphism $\sigma: \mathbb{H} \rightarrow \mathbb{H}$ that takes $I_{1}$ to $i$. By the Skolem-Noether theorem, it is of the form $\sigma(h)=a h a^{-1}$, so $\sigma\left(f_{1}(x)\right)=a f_{2}(x) a^{-1}$ for every $x \in \mathbb{H}$.

\section{Fueter Regular Functions}

Definition 11. Let $f: \mathbb{H} \rightarrow \mathbb{H}$ be a real differentiable function. The differential of $f$ at each $q \in \mathbb{H}$ is an $\mathbb{R}$-linear map $d_{f}: \mathbb{H} \rightarrow \mathbb{H}$

$$
d f=\frac{\partial f}{\partial x_{0}} d x_{0}+\frac{\partial f}{\partial x_{1}} d x_{1}+\frac{\partial f}{\partial x_{2}} d x_{2}+\frac{\partial f}{\partial x_{3}} d x_{3} .
$$

For example, the differential of the identity function is

$$
d q=d x_{0}+i d x_{1}+j d x_{2}+k d x_{3} .
$$

The exterior product of $d q$ with itself is

$$
d q \wedge d q=i d x_{2} \wedge d x_{3}+j d x_{3} \wedge d x_{1}+k d x_{1} \wedge d x_{2}
$$

Consider the following quaternionic valued 3-form

$$
D_{q}=d x_{1} \wedge d x_{2} \wedge d x_{3}-i d x_{0} \wedge d x_{2} \wedge d x_{3}+j d x_{0} \wedge d x_{1} \wedge d x_{3}+k d x_{0} \wedge d x_{1} \wedge d x_{2} .
$$


Lemma 2 (Poincaré-Stokes lemma). Let $\Omega$ be a simply connected domain in $\mathbb{H}$ with smooth boundary $\partial \Omega$, and let $W$ be a real differentiable 3-form. Then

$$
\int_{\partial \Omega} W=\int_{\Omega} d W
$$

Definition 12. A real differentiable function $f: U \subset \mathbb{H} \rightarrow \mathbb{H}$ is right Fueter-regular if and only if

$$
\partial_{r}(f)=\frac{\partial f}{\partial x_{0}}-\frac{\partial f}{\partial x_{1}} i-\frac{\partial f}{\partial x_{2}} j-\frac{\partial f}{\partial x_{3}} k=0,
$$

and it is left Fueter regular if and only if

$$
\partial_{l}(f)=\frac{\partial f}{\partial x_{0}}-i \frac{\partial f}{\partial x_{1}}-j \frac{\partial f}{\partial x_{2}}-k \frac{\partial f}{\partial x_{3}}=0 .
$$

\section{Structural Regular Functions}

The class of Fueter-regular functions does not contain many of the most important functions such as power series, polynomials, or even the identity. We work with another class of regular functions, [8] defined by the Cauchy-Riemann-Fueter operator associated with the structural vector $\{1, i, j,-k\}$. Indeed, for every $q \in \mathbb{H}, q=z_{1}+z_{2} j=x_{0}+i x_{1}+j x_{2}+k x_{3}$, where $\left(z_{1}, z_{2}\right)=\left(x_{0}+i x_{1}, x_{2}+i x_{3}\right)$. A quaternionic function $f=f_{1}+f_{2} j \in C^{1}(\Omega)$ is left structural regular on $\Omega$ if

$$
\mathcal{D}^{\prime} f=\frac{\partial f}{\partial x_{0}}+i \frac{\partial f}{\partial x_{1}}+j \frac{\partial f}{\partial x_{2}}-k \frac{\partial f}{\partial x_{3}}=0 \text { on } \Omega,
$$

and it is right structural regular if

$$
\mathcal{D}^{\prime} f=\frac{\partial f}{\partial x_{0}}+\frac{\partial f}{\partial x_{1}} i+\frac{\partial f}{\partial x_{2}} j-\frac{\partial f}{\partial x_{3}} k=0 \text { on } \Omega .
$$

We will denote by $\mathcal{R}(\Omega)$ the space of all structural regular functions on $\Omega$. By this definition, the space $\mathcal{R}(\Omega)$ contains the identity mapping, which is a bigger class than Fueter regular functions.

\section{Cauchy's Theorem and Integral Formula}

The integral theorem for regular complex functions can be generalized to the regular quaternionic functions.

Proposition 1. Let $\Omega$ be a bounded simply connected domain in $\mathbb{H}$, and $\partial \Omega$ is its smooth boundary. Let $f$ be a differentiable quaternion-valued function in an open neighbourhood of $\Omega$, and $\mathcal{D}^{\prime} f=\frac{\partial f}{\partial x_{0}}+i \frac{\partial f}{\partial x_{1}}+j \frac{\partial f}{\partial x_{2}}-k \frac{\partial f}{\partial x_{3}}$. Then

$$
\int_{\partial \Omega} D_{x} f(x)=\int_{\Omega}\left[\mathcal{D}^{\prime} f(x)\right] d x_{0} \wedge d x_{1} \wedge d x_{2} \wedge d x_{3} .
$$

Moreover, if $f$ is a left structural regular function in an open neighbourhood of $D$, then

$$
\int_{\partial D} D_{x} f(x)=0
$$


Proof. Equation (7) is a direct consequence of the definition of a left structural regular function (4) and Equation (6). Equation (6) is a consequence of the the Poincaré-Stokes lemma. Indeed, let $W=D_{x} f(x)$. Then

$$
\begin{aligned}
d W & =d x_{0} \wedge \partial_{x_{0}}\left(D_{x} f(x)\right)+d x_{t} \wedge \partial_{x_{t}}\left(D_{x} f(x)\right) \\
& =d x_{0} \wedge d x_{1} \wedge d x_{2} \wedge d x_{3} f_{x_{0}}-d x_{1} \wedge d x_{0} \wedge d x_{2} \wedge d x_{3} i f_{x_{1}} \\
& +d x_{2} \wedge d x_{0} \wedge d x_{1} \wedge d x_{3} j f_{x_{2}}+d x_{3} \wedge d x_{0} \wedge d x_{1} \wedge d x_{2} k f_{x_{3}} \\
& =d x_{0} \wedge d x_{1} \wedge d x_{2} \wedge d x_{3} f_{x_{0}}+d x_{0} \wedge d x_{1} \wedge d x_{2} \wedge d x_{3} i f_{x_{1}} \\
& +d x_{0} \wedge d x_{1} \wedge d x_{2} \wedge d x_{3} j f_{x_{2}}-d x_{0} \wedge d x_{1} \wedge d x_{2} \wedge d x_{3} k f_{x_{3}} \\
& =\left(f_{x_{0}}+i f_{x_{1}}+j f_{x_{2}}-k f_{x_{3}}\right) d x_{0} \wedge d x_{1} \wedge d x_{2} \wedge d x_{3} \\
& =\left[\mathcal{D}^{\prime} f(x)\right] d x_{0} \wedge d x_{1} \wedge d x_{2} \wedge d x_{3} .
\end{aligned}
$$

Then (6) follows.

Similarly, this proposition is true for a differentiable quaternion-valued function that is right regular:

$$
\int_{\partial D} f(x) D_{x}=0
$$

Proposition 2. Let $\Omega$ be a bounded simply connected domain in $\mathbb{H}$ with a smooth boundary $\partial \Omega$. Let $f$ and $g$ be differentiable quaternion-valued functions in an neighborhood of $\Omega$. Then

$$
\int_{\partial \Omega} g(x) D_{x} f(x)=\int_{\Omega}\left[\left(\mathcal{D}^{\prime} g(x)\right) f(x)+g(x)\left(\mathcal{D}^{\prime} f(x)\right)\right] d x_{0} \wedge d x_{1} \wedge d x_{2} \wedge d x_{3} .
$$

Moreover, if $g$ is a right structural regular function and $f$ is a left structural regular function, then

$$
\int_{\partial \Omega} g(x) D_{x} f(x)=0
$$

Taking $g=1$, we obtain Proposition 4.1.

\section{A New Analogue of the Cauchy Integral Formula}

Let $U \subset \mathbb{C}$ be an open set with boundary $\partial U$ consisting of a finite number of rectifiable Jordan curves. If $f$ is a complex-valued function that is holomorphic on an open set that contains $\bar{U}$, then Cauchy's integral formula is

$$
f(z)=\frac{1}{2 \pi i} \int_{\partial U} \frac{f\left(z^{\prime}\right)}{z^{\prime}-z} d z^{\prime},
$$

for any $z \in U$. Therefore, in order to define an analogous Cauchy's integral formula for analytic quaternionic-valued functions we need to construct the quaternionic Cauchy kernel $\left(q-q_{0}\right)^{-1}$. The first option is the function $\frac{\left(q-q_{0}\right)^{-1}}{\left|q-q_{0}\right|^{2}}$, which is both a Fueter-right and left-regular function at each $q \neq q_{0}$. However, it is not a structural regular function. Now consider the Cauchy kernel

$$
K(p, q)=\frac{\left[\left(p_{0}-q_{0}\right)+i\left(p_{1}-q_{1}\right)+j\left(p_{2}-q_{2}\right)-k\left(p_{3}-q_{3}\right)\right]^{-1}}{|p-q|^{2}},
$$

where $p=p_{0}+i p_{1}+j p_{2}+k p_{3}$ and $q=q_{0}+i q_{1}+j q_{2}+k q_{3}$. This new Cauchy kernel is both right and left structural regular. Therefore, using this function, we define the analogue of the Cauchy integral formula for the quaternionic case. 
Proposition 3. [7] Let $D$ be a bounded simply connected domain in $\mathbb{H}$ with a smooth boundary $\partial D$. Let $f$ : $U \subset \mathbb{H} \rightarrow \mathbb{H}$, where $U$ is an open neighborhood of $D$ and $f$ is left structural regular. Let $q_{0}$ be a point in the interior of $U$. Then

$$
f(q)=\frac{1}{2 \pi^{2}} \int_{\partial D} K(p, q) D_{p} f(p)
$$

\section{S-Resolvent Operator and S-Spectrum}

Let $p=p_{0}+i p_{1}+j p_{2}+k p_{3}$. Consider a real involutive linear map $J: \mathbb{H} \rightarrow \mathbb{H}$, so that $J(1)=1$, $\mathrm{J}(\mathrm{i})=\mathrm{i}, \mathrm{J}(\mathrm{j})=\mathrm{j}$, and $\mathrm{J}(\mathrm{k})=-\mathrm{k}$, for example, $\mathrm{J} p:=p_{0}+i p_{1}+j p_{2}-k p_{3}$. Let $T: X \rightarrow X$ be a $\mathbb{H}-$-linear operator on a $\mathbb{H}-C^{*}$-algebra $X$ and $I$ be the identity operator on $X$. Then we define

$$
J(T-q I):=\left(T_{0}-q_{0} I\right)+i\left(T_{1}-q_{1} I\right)+j\left(T_{2}-q_{2} I\right)-k\left(T_{3}-q_{3} I\right),
$$

where $T_{i}: X_{R e} \rightarrow X_{R e}$ for $i=0,1,2$, and 3 are real linear operators on $X_{R e}=\{x \in X: x \alpha=\alpha x\}$, the real part of $X$.

The following theorem seems to be new, and in fact, the nice properties of the map $J$ appear to not have been noticed.

Theorem 7. Let $X$ be a $\mathbb{H}-C^{*}$-algebra, $T$ be a self-adjoint $\mathbb{H}-$ linear operator on $X$, and let $q \in \mathbb{H}$. Then

$$
T^{*} q+\bar{q} T=2 \operatorname{Re}\left[J T \bar{q} J^{*}\right]=2 \operatorname{Re}\left[J q T^{*} J^{*}\right] .
$$

Moreover, suppose that $(J(T-q I))^{*}$ is invertible. Then

$$
(J(T-q I))=\left(T^{*} T-2 \operatorname{Re}\left[J T \bar{q} J^{*}\right]+|q|^{2} I\right)\left[(J(T-q I))^{*}\right]^{-1} .
$$

Proof. By evaluation, we have

$$
\begin{aligned}
(J(T-q I))^{*}(J(T-q I))= & \left(\left(T^{*}-\bar{q} I\right) J^{*}\right)(J(T-q I)) \\
& =T^{*} J^{*} J T-T^{*} J^{*} J q I-\bar{q} I J^{*} J T+\bar{q} I J^{*} J q I \\
& =T^{*} T-\left[T^{*} q+\bar{q} T\right]+|q|^{2} I,
\end{aligned}
$$

and

$$
\begin{aligned}
(J(T-q I))(J(T-q I))^{*}= & (J(T-q I))\left(\left(T^{*}-\bar{q} I\right) J^{*}\right) \\
& =J T T^{*} J^{*}-J T \bar{q} I J^{*}-J q I T^{*} J^{*}+J q I \bar{q} I J^{*} \\
& =T^{*} T-2 \operatorname{Re}\left[J T \bar{q} J^{*}\right]+|q|^{2} I .
\end{aligned}
$$

Therefore, from (12) and (13), $T^{*} q+\bar{q} T=2 \operatorname{Re}\left[J T \bar{q} J^{*}\right]=2 \operatorname{Re}\left[J q T^{*} J^{*}\right]$, and we have

$$
(J(T-q I))=\left(T^{*} T-2 \operatorname{Re}\left[J T \bar{q} J^{*}\right]+|q|^{2} I\right)\left[(J(T-q I))^{*}\right]^{-1} .
$$

Definition 13 (The $S$-resolvent operators). Let $T$ be a self-adjoint operator on a $\mathbb{H}-C^{*}$-algebra $X$, then define the S-resolvent operator, following [3], as

$$
S^{-1}(q, T):=(J(T-(q I)))^{*}\left(T^{*} T-2 \operatorname{Re}\left(J T \bar{q} J^{*}\right)+|q|^{2} I\right)^{-1} .
$$

Though S-resolvents have been studied, the above way of writing them in terms of $J$ may be new. 
Definition 14 (The spectra of quaternionic operators). Let $T: X \rightarrow X$ be a self-adjoint operator on a $\mathbb{H}-C^{*}$-algebra $X$. The left spectrum of $T$ is defined by

$$
\sigma_{L}(T)=\{q \in \mathbb{H}: T-q I \text { is not invertible }\},
$$

and define the S-spectrum of $T$ related to the S-resolvent operator (14) as

$$
\sigma_{S}(T)=\left\{q \in \mathbb{H}:\|T\|^{2}-2 \operatorname{Re}\left(J T \bar{q} J^{*}\right)+|q|^{2} I \text { is not invertible }\right\} .
$$

\section{Functional Calculus}

Let $f$ be a left structural regular function and $T$ be a self-adjoint operator on a $\mathbb{H}-C^{*}$-algebra $X$. We define

$$
f(T)=\frac{1}{2 \pi^{2}} \int_{\partial \Omega} \frac{S^{-1}(q, T)}{|J(T-q I)|^{2}} D_{q}, f(q)
$$

where $q \in \sigma_{S}(T)$ and $\Omega$ is an open bounded set that contains $\sigma_{S}(T)$.

Theorem 8. Let $q^{m}$ a be a monomial, which is left structural regular for some $m \in \mathbb{N} \cup\{0\}, q, a \in \mathbb{H}$. Let $T$ be a self-adjoint operator on $a \mathbb{H}-C^{*}$-algebra $X$, and let $\Omega$ be an open bounded set that contains $\sigma_{S}(T)$. Then

$$
T^{m} a=\frac{1}{2 \pi^{2}} \int_{\partial \Omega} \frac{S^{-1}(q, T)}{|J(T-q I)|^{2}} D_{q} q^{m} a
$$

Proof. Let $S$ be a 3 - sphere with center at $q$ on which

$$
D_{q}=\frac{J(T-q I)}{|J(T-q I)|} d S,
$$

where $S$ is the 3 -sphere. Hence

$$
\begin{aligned}
\frac{1}{2 \pi^{2}} \int_{\partial \Omega} \frac{S^{-1}(q, T)}{|J(T-q I)|^{2}} D_{q} q^{m} a & =\frac{1}{2 \pi^{2}} \int_{S} \frac{S^{-1}(q, T)}{|J(T-q I)|^{2}} \frac{J(T-q I)}{|J(T-q I)|} d S q^{m} a \\
& =\frac{1}{2 \pi^{2}} \int_{S} \frac{\left.(J(T-(q I)))^{*}\left(\mid T^{*} T-2 R e\left[J T \bar{q} J^{*}\right)\right]+|q|^{2} I\right)^{-1}(J(T-q I))}{|J(T-q I)|^{3}} d s f(T) \\
& =\frac{1}{2 \pi^{2}} \int_{S} \frac{(J(T-(q I)))^{*}\left[\left(J(T-(q I))^{*}\right)^{-1}\left(J(T-(q I))^{-1}\right](J(T-q I))\right.}{|J(T-q I)|^{3}} d s T^{m} a \\
& =\frac{1}{2 \pi^{2}} \int_{S} \frac{d S}{|J(T-q I)|^{3}} T^{m} a \\
& =\frac{1}{2 \pi^{2}} 2 \pi^{2} \frac{|J(T-q I)|^{3}}{|J(T-q I)|^{3}} T^{m} a \\
& =T^{m} a .
\end{aligned}
$$

Author Contributions: The Authors contribute equally to this work.

Funding: This research received no external funding

Conflicts of Interest: The authors declare no conflict of interest.

\section{References}

1. Von Neumann, J. Eine Spektraltheorie fur allgemeine Operatoren eines unitaren Raumes. Math. Nachr. 1951, $4,258-281$. 
2. Gentili, G.; Struppa, D.C. A new approach to Cullen-regular functions of a quaternionic variable. C. R. Acad. Sci. Paris 2006, 342, 741-744. [CrossRef]

3. Ghiloni, R.; Moretti, V.; Perotti, A. Continuous slice functional calculus in quater-nionic Hilbert spaces. Rev. Math. Phys. 2013, 25, 1350006. [CrossRef]

4. Gelfand, I.; Raikov, D.; Shilov, G. Commutative Normed Rings; Translated 1964; Chelsea Publishing Co.: New York, NY, USA, 1960; ISBN 978-0-8218-2022-3.

5. Ng, C.-K. On quaternionic functional analysis. Math. Proc. Camb. Phil. Soc. 2008, 143, 391-406. [CrossRef]

6. Kulkarni, S.H. Representations of a class of real B*-algebras as algebras of quaternion-valued functions. Proc. Am. Math. Soc. 1992, 116, 61-66.

7. Sudbury, T. Quaternionic Analysis. In Mathematical Proceedings of the Cambridge Philosophical Society; Cambridge University Press: Cambridge, UK, 1979; Volume 85.

8. Perotti, A. Quaternionic regularity and the $\partial$-Neumann problem in $\mathbb{C}$, to appear on Complex Variables and Elliptic Equations. arXiv 2006, arXiv:math/0612092.

(C) 2019 by the authors. Licensee MDPI, Basel, Switzerland. This article is an open access article distributed under the terms and conditions of the Creative Commons Attribution (CC BY) license (http:/ / creativecommons.org/licenses/by/4.0/). 